\title{
Silent myocardial dysfunction in vitamin D deficiency
}

Pelin Karaca Ozer, Samim Emet, Ekrem Bilal Karaayvaz, Ali Elitok, Ahmet Kaya Bilge, Kamil Adalet, Aytac Oncul

Department of Cardiology, Medical Faculty, Istanbul University, Istanbul, Turkey

Submitted: 26 April 2020

Accepted: 28 May 2020

Arch Med Sci Atheroscler Dis 2020; 5: e153-e162

DOI: https://doi.org/10.5114/amsad.2020.97110

Copyright @ 2020 Termedia \& Banach

\section{Abstract}

Introduction: Vitamin D (VD) deficiency is a common disease that occurs in all stages of life. A growing number of studies call attention to the relationship between VD deficiency and cardiovascular disease. The aim of this study was to investigate the effect of VD on subclinical left ventricular (LV) function in diabetic and non-diabetic patients with no significant coronary artery disease.

Material and methods: We recruited 140 patients ( 80 diabetics and 60 nondiabetics) with symptoms of stable ischemic heart disease who underwent coronary angiography and who had no significant coronary artery disease in our clinic. The $25(\mathrm{OH}) \mathrm{D}_{3}$ levels were measured and patients who had $25-(\mathrm{OH}) \mathrm{D}_{3}$ levels below $20 \mathrm{ng} / \mathrm{dl}$ were defined as the VD deficient group. In addition to conventional echocardiographic parameters, tissue Doppler echocardiography was used for LV diastolic functions and 2D speckle tracking strain echocardiography (2D STE) for evaluating the longitudinal deformation indices of the LV myocardium.

Results: In all groups, LV global longitudinal strain (GLS) was significantly impaired in patients with VD deficiency $(p<0.001)$ compared to patients without VD deficiency. LV global longitudinal strain rate (GLSR) was significantly impaired in patients with VD deficiency $(p=0.003)$. The GLS was negatively associated with $25-(\mathrm{OH}) \mathrm{D}_{3}$ in the VD deficiency group $(r=-0.52623$, $p<0.001)$. Conversely, GLS was positively associated with $25-(\mathrm{OH}) \mathrm{D}_{3}$ levels in the normal VD group $(r=0.28, p=0.048)$.

Conclusions: VD deficiency is associated with impaired myocardial GLS. The present study demonstrated that VD deficiency may be the cause of subclinical myocardial dysfunction in patients with or without diabetes mellitus and no history of significant coronary artery disease.

Key words: vitamin D, myocardial deformation, strain, strain rate, coronary artery disease.

\section{Introduction}

Vitamin D (VD) is a steroid hormone and regulates calcium and bone metabolism. VD also plays a role in several other biochemical mechanisms within the body [1, 2]. The mechanism of action of the active form of VD is mediated by the VD receptor (VDR). VDRs are found in cardiomyocytes [3, 4].

Serum VD deficiency is a common condition in population-wide studies. Epidemiologic studies have shown that VD deficiency was associated with chronic diseases such as cancer, type 2 diabetes, autoimmune diseases and cardiovascular disease (hypertension, coronary artery disease,

\author{
Corresponding author: \\ Samim Emet MD \\ Department of Cardiology \\ Medical Faculty \\ Istanbul University \\ Istanbul, Turkey \\ Phone: +902124142000 \\ E-mail: samim.emet@ \\ istanbul.edu.tr
}


peripheral artery disease, heart failure and stroke) $[5,6]$. Thus, VD deficiency can be a cardiovascular risk factor and associated with cardiovascular morbidity and mortality $[7,8]$.

VD has been related to some pathological changes in the myocardium in previous studies [9-11]. However, the effect of VD deficiency on the deformation parameters of the myocardium is very limited. Conventional echocardiographic parameters such as LV ejection fraction (LVEF) are not sensitive enough to detect subclinical cardiac systolic dysfunction. The 2D STE is a relatively novel and sensitive echocardiographic modality that can assess regional and/or globally subtle LV myocardial impairment with strain and strain rate measurements [12]. The aim of the present study was to evaluate the association of VD deficiency and longitudinal deformation of the LV myocardium measured by 2D STE in diabetic and non-diabetic patients without significant coronary artery disease.

\section{Material and methods}

The present study was an observational cross-sectional study, including 140 patients who were admitted to the Istanbul Faculty of Medicine, Department of Cardiology with symptoms of stable ischemic heart disease in the period 2014-2019. The patients underwent coronary angiography and none of them had obstructive coronary artery disease. Obstructive coronary artery disease was defined by the presence of a plaque with $50 \%$ or greater stenosis on epicardial coronary arteries. Age, sex, body mass index (BMI), coronary artery disease risk factors (hypertension, diabetes mellitus, dyslipidemia, smoking) and the usage of cardio-protective drugs were recorded. Blood samples were obtained for measurement of $25(\mathrm{OH}) \mathrm{D}_{3}$. All patients were evaluated with conventional echocardiographic parameters, tissue Doppler echocardiography, and 2D STE to assess left ventricular function.

The study was approved by the local ethics committee. All patients gave informed consent. Exclusion criteria were previous myocardial infarction, cardiomyopathies, EF less than $50 \%$, severe valvular disease, chronic renal and liver disease, active malignancies, sustained supraventricular or ventricular arrhythmias during angiography, and the usage of VD replacement therapy.

\section{Laboratory method}

To avoid seasonal variations, all blood samples were collected during winter (November-February). Blood samples were stored at $-70^{\circ} \mathrm{C}$ within 30 min of collection. Serum $25(\mathrm{OH}) \mathrm{D}_{3}$ concentrations were measured using an automated Vitamin
D2-D3 HPLC Analyzer (Zivak Technologies 25-OH Vitamin D2-D3 HPLC analysis kit). The intra- and inter-assay coefficients of variation were $7 \%$ and $11 \%$, respectively. Patients were categorized into two groups as vitamin D deficient $<20 \mathrm{ng} / \mathrm{ml}$ and vitamin D sufficient $\geq 20 \mathrm{ng} / \mathrm{dl}$ ) according to Endocrine Society guidelines [13].

\section{Echocardiography}

One experienced observer performed echocardiography on all subjects with an iE33xMATRIX ultrasound system (Philips Medical Systems, Andover, Massachusetts) with an X5-1 (1-5 MHz) transducer using second harmonic imaging. Conventional echocardiographic measurements were performed in accordance with the recommendations of the American Society of Echocardiography guidelines [14]. Measurements were obtained with parasternal and apical views. The LV dimensions used an average of five M-mode measurements and included diastolic and systolic diameters, diastolic thickness of the interventricular septum and diastolic posterior wall thickness. Systolic function of the LV was determined as fractional shortening and EF was determined via two-dimensional M-mode.

LV diastolic function was assessed using pulsed Doppler measurements of filling in the apical four-chamber view. The Doppler beam was aligned perpendicularly to the mitral annulus plane and the sample volume was placed between the tips of the mitral leaflets. Five consecutive beats of mitral valve inflow were collected to calculate peak early diastolic filling (E wave) and late diastolic filling (A wave) velocities, the E/A ratio, and deceleration time (DT) of the E wave. Early diastolic ( $\left.\mathrm{e}^{\prime}\right)$ and late diastolic $\left(a^{\prime}\right)$ velocities were measured in the septal and lateral wall using a $5 \mathrm{~mm}$ circular sample volume placed in the left ventricular myocardial just proximal to the mitral annular level and the mean of septal and lateral $e^{\prime}-a^{\prime}$ velocities accepted. The isovolumic relaxation time (IVRT), isovolumic contraction time (IVCT) and ejection time (ET) values were determined [15]. Myocardial performance index (MPI) is an index of global ventricular function defined as the sum of IVCT and IVRT divided by ET [16]. All size and mass indices were indexed to body surface area.

\section{D Speckle Tracking Strain Imaging}

Strain was defined as the instantaneous local trace lengthening or shortening and strain rate was the rate of lengthening or shortening [16]. Speckle tracking strain imaging showed three consecutive cardiac cycles from the apical 4-, 3- (long axis), and 2-chamber views. These were obtained at 42-56 frames per second and then stored. The aCMQ option of the QLAB 10.8.5 software was 
used for deformation analysis. First, for each view, the operator placed three points (two points at the base of the LV and one point at the apex) at the end of diastole. The endocardial and epicardial borders were then automatically traced by the software. Adjustments were made by the operator if required. Systolic strain and strain rate values were calculated at aortic valve closure time by averaging the values of all segments for assessment of LV global longitudinal systolic function [17].

Each wall of the LV was segmented into three (base, mid and apical) equal parts automatically and 18 segmental strain curves were obtained (Figure 1) to give the so-called bull's-eye plots (Figure 2). Segments with poor tracking despite manual readjustments of the region of interest were excluded. Healthy individuals had an average peak systolic LV longitudinal strain as assessed by speckle tracking from -18 to $-20 \%[17,18]$. The global longitudinal strain (GLS) was determined to be reduced or normal (Figures 1, 2).

\section{Statistical analysis}

Statistical analysis was performed with SPSS 19.0 for Windows (IBM Corp. since v. 19.0, Chicago, IL, USA). Continuous data are expressed as the mean $\pm S D$, categorical data are expressed as percentages. The $\chi^{2}$-test was used to assess differences between categorical variables among the groups. The relationships among parameters were assessed using Pearson correlation analysis. Student's $t$-test compared unpaired samples. Logistic regression analyses of strain and strain rate by vitamin $\mathrm{D}$ insufficiency and traditional cardiac risk factors (age, sex, smoking, DM, HT, and $\mathrm{HL}$ ) were evaluated among patients. A $p$-value $<0.05$ was considered statistically significant.

\section{Results}

\section{Clinical characteristics}

We enrolled 140 patients with 80 diabetics and 60 non-diabetics. The gender ratio and cardiovascular risk factors were similar in both groups. Diabetic patients had a lower glomerular filtration rate (GFR) (77.96 \pm 19.06 ; $94.08 \pm 26.10$; $p<0.001)$ and a higher BMI (25.99 $\pm 1.77 ; 23.89$ $\pm 2.8 ; p<0.001$ ). Cardiovascular drug usage such as angiotensin converting enzyme inhibitors ( $p<0.001)$, $\beta$-blocker $(p=0.031)$ and statins $(p=0.005)$ were higher in the diabetic group (Table I). Insulin therapy was prescribed to 38 (48\%) patients and these patients had a higher $\mathrm{HbA}_{1 \mathrm{c}}$ level $(8.1 \pm 1.1$ vs. $7.0 \pm 1.1 \%, p<0.01)$ than patients not taking insulin therapy.

The average $25(\mathrm{OH}) \mathrm{D}_{3}$ level for all groups was $16.2 \mathrm{ng} / \mathrm{ml}$ (range: $1.6-48,8 \mathrm{mg} / \mathrm{dl}$ ). The prevalence of VD deficiency in the diabetic group was
$68 \%$ and $63 \%$ in the non-diabetic group ( $p=$ 0.456). Patients with VD deficiency were more likely to be female. There was no significant difference in age or cardiovascular risk factors between the two groups (Table II).

\section{Echocardiographic parameters}

Conventional echocardiographic parameters of LV systolic and diastolic function in diabetic and non-diabetic patients are presented in Table III. There were no significant differences in LV volumes, EF, wall thickness or LV mass index. In the diabetic group, diastolic function parameters were significantly impaired. The MPI was significantly increased in the diabetic group $(p<0.001)$. The LV GLS was significantly impaired in diabetic patients versus the non-diabetic group $(t=2.353$, $p=0.020$ ). The LV global longitudinal strain rate (GLSR) was significantly impaired in diabetics versus non-diabetics ( $t=2.461, p=0.030)$.

Conventional echocardiographic parameters of LV systolic and diastolic function in patients with and without VD deficiency are presented in Table IV. There were no significant differences in LV volumes, EF, wall thickness, LV mass index, diastolic grade, E-E' velocity, DT, IVRT or MPI in patients with and without VD deficiency. The LV GLS was significantly impaired in patients with VD deficiency $(p<0.001)$ versus those without VD deficiency. The LV GLSR was significantly impaired in patients with VD deficiency $(p=0.003)$.

Among the diabetic group, VD deficiency is significantly associated with impaired GLS ( $p<$ $0.001)$ and GLSR ( $p=0.004)$. However, there was no significant effect of VD on diastolic function parameters or MPI in diabetics.

Among the non-diabetic group, VD deficiency was associated with impaired GLS ( $p=0.049)$. GLSR was not significantly different between those with and without VD deficiency $(p=0.060)$. Again, there was no significant effect of VD on diastolic function parameters or MPI in non-diabetics. The correlation of VD and LV GLS is presented in Figure 3.

GLS was negatively associated with the level of $25(\mathrm{OH}) \mathrm{D}_{3}$ in the VD deficient group $(r=-0.52623$, $p<0.001)$. Conversely, GLS was positively associated with the level of $25(\mathrm{OH}) \mathrm{D}_{3}$ in the VD sufficient group $(r=0.286465, p=0.048)$. Logistic regression analysis demonstrated that LV GLS was independently associated with VD deficiency, hypertension, triglyceride levels, age and BMI. GLS was positively associated with age $(r=0.22, p=$ $0.010)$, BMI $(r=0.26, p=0.002)$ and triglyceride levels $(r=0.19, p=0.020)$. There was no correlation between GLS and insulin therapy $(t=1.663$, $p=0.098)$ or $\mathrm{HbA}_{1 c}$ levels $(r=0.041, p=0.714)$ in the diabetic group. 

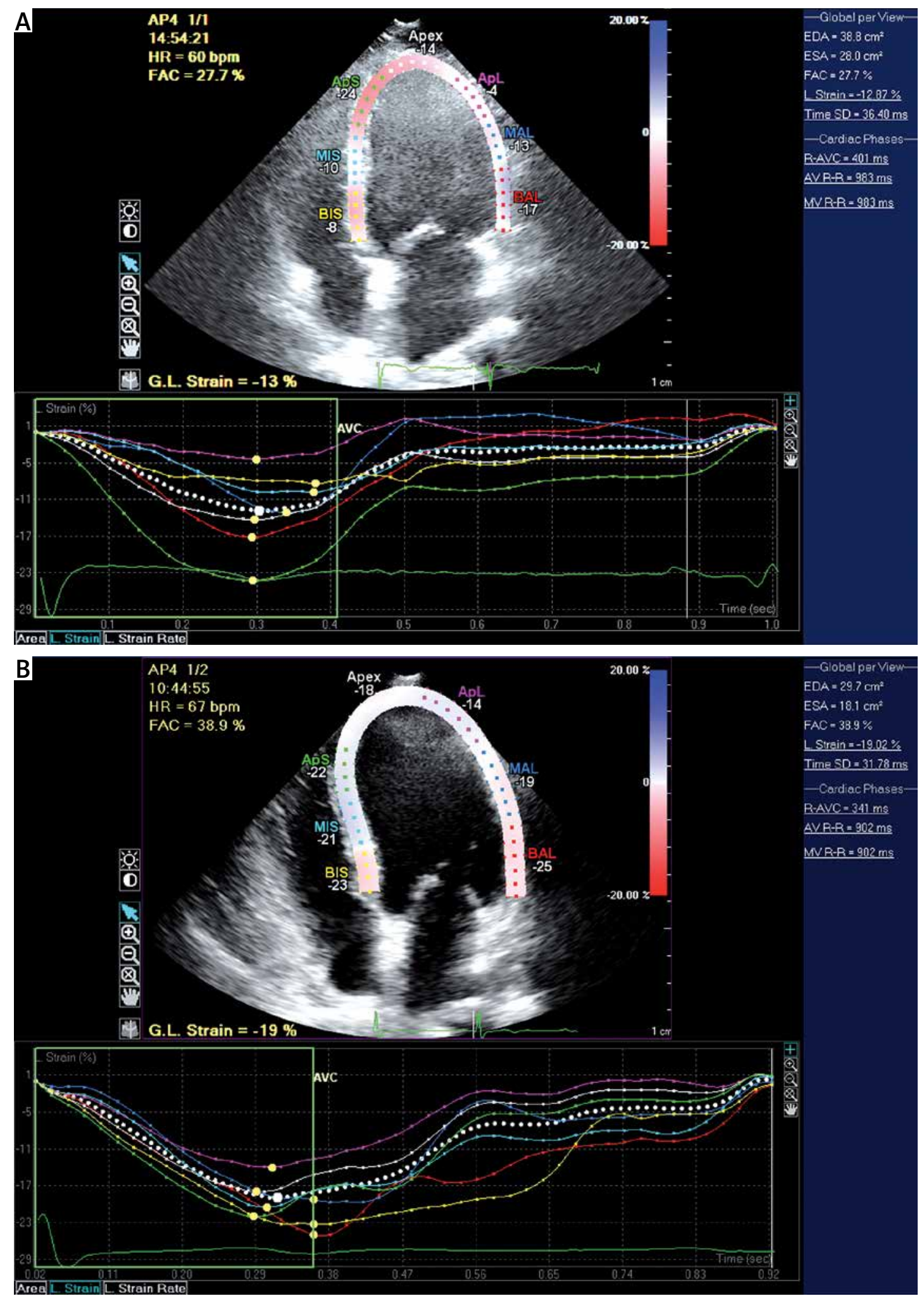

Figure 1. A - Strain curves showing impaired global longitudinal strain in apical 4-chamber view. B - Strain curves showing normal global longitudinal strain in apical 4-chamber view 

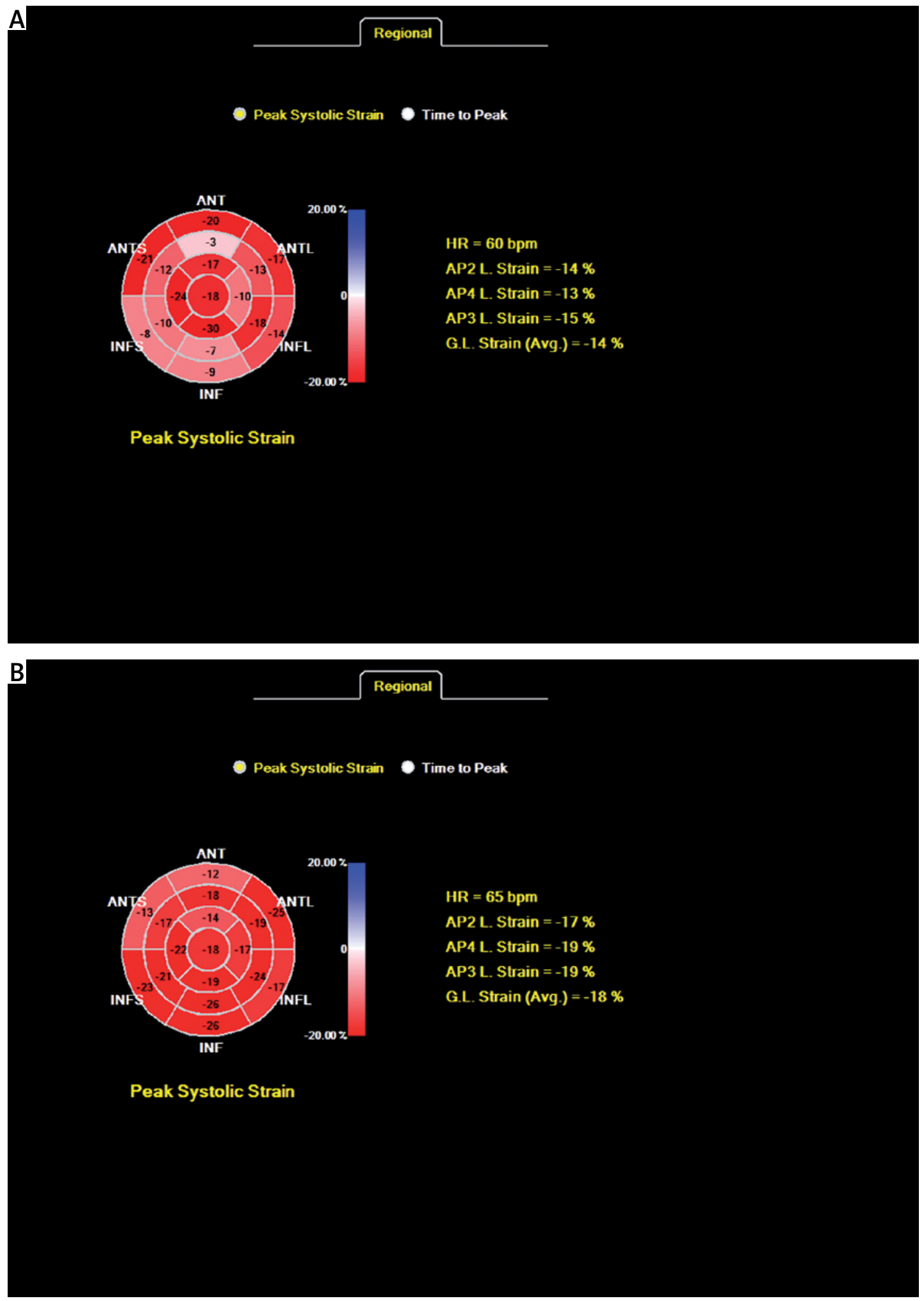

Figure 2. A - A bull's-eye plot showing impaired global longitudinal strain in patients with vitamin D deficiency. B - A bull's-eye plot showing normal global longitudinal strain in patients without vitamin D deficiency 
Table I. Clinical characteristics of diabetic and non-diabetic patients

\begin{tabular}{|c|c|c|c|}
\hline Parameter & Diabetic group $(n=80)$ & Non-diabetic group $(n=60)$ & $P$-value \\
\hline Age [years] & $57.9 \pm 6.26(43-70)$ & $50.9 \pm 12.01(32-70)$ & 0.56 \\
\hline Male, $n(\%)$ & $35(43.8)$ & $28(46.7)$ & 0.73 \\
\hline BMI & $25.99 \pm 1.77(21.8-29.8)$ & $23.89 \pm 2.8(20.1-28.7)$ & $<0.001$ \\
\hline Hypertension, $n(\%)$ & $31(38.8)$ & $19(31.7)$ & 0.38 \\
\hline Current smoker, $n(\%)$ & $32(40)$ & $29(48.3)$ & 0.32 \\
\hline $\mathrm{SBP}[\mathrm{mm} \mathrm{Hg}]$ & $123.89 \pm 9.99(100-140)$ & $124.56 \pm 11.601(95-140)$ & 0.35 \\
\hline $\mathrm{DBP}[\mathrm{mm} \mathrm{Hg}]$ & $70.689 \pm 10.24(60-90)$ & $69.490 \pm 10.83(60-90)$ & 0.78 \\
\hline Heart rate $[\mathrm{bpm}]$ & $68.96 \pm 8.61(55-92)$ & $71.31 \pm 7.26(58-87)$ & 0.057 \\
\hline eGFR $\left[\mathrm{ml} / \mathrm{min} / \mathrm{m}^{2}\right]$ & $77.96 \pm 19.06(48-151)$ & $94.08 \pm 26.1(49-156)$ & $<0.001$ \\
\hline \multicolumn{4}{|c|}{ Serum lipid profile [ $\mathrm{mg} / \mathrm{dl}]$ : } \\
\hline Total cholesterol & $192.81 \pm 39.02(119-268)$ & $221.5 \pm 50.4(162-373)$ & 0.051 \\
\hline LDL & $131.95 \pm 32.1(57-210)$ & $133.91 \pm 40.8(32-244)$ & 0.97 \\
\hline Triglycerides & $199.02 \pm 88.1(54-523)$ & $175.1 \pm 73.8(82-363)$ & 0.09 \\
\hline $\mathrm{HDL}$ & $41.23 \pm 10.7(23-74)$ & $45.54 \pm 16.02(24-121)$ & 0.14 \\
\hline \multicolumn{4}{|c|}{ Medical treatment, $n(\%)$ : } \\
\hline ACEI & $38(47.5)$ & $9(15)$ & $<0.001$ \\
\hline ARB & $19(23.8)$ & $7(11.7)$ & 0.06 \\
\hline$\beta$-blockers & $37(46.3)$ & $17(28.3)$ & 0.03 \\
\hline CCB & $15(18.8)$ & $8(13.3)$ & 0.39 \\
\hline Statin & $43(53.8)$ & $8(30)$ & 0.005 \\
\hline \multicolumn{4}{|l|}{ Serum biomarkers: } \\
\hline $25-\mathrm{OHD}[\mathrm{ng} / \mathrm{ml}]$ & $16.08 \pm 10.89(2.2-42.6)$ & $15.42 \pm 10.31(1.6-48.8)$ & 0.45 \\
\hline $\mathrm{CRP}[\mathrm{mg} / \mathrm{ll}]$ & $4.94 \pm 4.4(0.1-18)$ & $4.21 \pm 3.8(0.3-19.3)$ & 0.46 \\
\hline
\end{tabular}

$B M I$ - body mass index, SBP - systolic blood pressure, DBP - diastolic blood pressure, eGFR - estimated glomerular filtration rate, ACEI angiotensin converting enzyme inhibitor, $A R B$ - angiotensin receptor blocker, CCB - calcium channel blocker, 25-OHD - 25 hydroxyvitamin D.

\section{Discussion}

It is estimated that most people worldwide are vitamin D deficient [19]. Recent studies demonstrated that VD deficiency was a significant risk factor for cardiovascular disease and associated with cardiovascular mortality $[20,21]$. The $25(\mathrm{OH}) \mathrm{D}_{3}$ levels are also associated with important cardiovascular disease risk factors such as diabetes mellitus, obesity, increased levels of triglycerides and hypertension [22-24].

The mechanism underlying the role of VD in the prevention of heart disease remains incompletely understood. However, the proposed mechanisms involve the presence of VDR in vascular smooth cells, endothelial cells and cardiomyocytes. VD can also affect cardiac function by altering parathormon (PTH) and serum calcium levels, regulation of hormone secretion, regulation of the renin-angiotensin-aldosterone (RAA) system, and regulation of blood pressure effects [25]. Activated 1,25(OH) , D suppresses renin gene expression in vitro and regulates the growth and proliferation of vascular smooth muscle cells and cardiomyocytes [25, 26]. VD induces pathological changes in the myocardium that are similar to pathological changes induced by diabetes [27]. Thus, these two conditions may lead to defects in myocardial deformation that are detectable by 2DSTE. The common pathways that cause myocardial damage include RAA activation, apoptosis and steatosis, leading to myocardial fibrosis, hypertrophy, lipid accumulation in the myocardium $[27,28]$, matrix metalloproteinase activation [28] and oxidative stress [29]. These features are seen in both diabetes and VD deficiency.

Collectively, these mechanisms may explain the relationship between VD deficiency and myocardial dysfunction although further evaluation is required. 
Table II. Clinical characteristics of patients with and without vitamin D (VD) deficiency

\begin{tabular}{|c|c|c|c|}
\hline Parameter & VD deficiency group $(n=92)$ & Group without VD deficiency $(n=48)$ & $P$-value \\
\hline Age [years] & $54.86 \pm 10.1(23-70)$ & $53.6 \pm 9.68(32-70)$ & 0.37 \\
\hline Male, $n(\%)$ & $41(45)$ & $22(46)$ & 0.88 \\
\hline BMI & $25.2 \pm 2.22(20.1-29.8)$ & $24.8 \pm 2.09(21.0-29.7)$ & 0.53 \\
\hline Hypertension, $n(\%)$ & $34(37)$ & $16(33)$ & 0.67 \\
\hline Type 2 DM, $n(\%)$ & $51(60)$ & $29(55)$ & 0.47 \\
\hline Current smoker, $n(\%)$ & $38(41)$ & $23(48)$ & 0.45 \\
\hline $\mathrm{SBP}[\mathrm{mm} \mathrm{Hg}]$ & $119.7 \pm 9.7(100-140)$ & $118.8 \pm 9.35(100-135)$ & 0.07 \\
\hline $\mathrm{DBP}[\mathrm{mm} \mathrm{Hg}]$ & $72.7 \pm 5.56(60-80)$ & $71.2 \pm 7.25(60-85)$ & 0.21 \\
\hline Heart rate $[\mathrm{bpm}]$ & $70.1 \pm 8.02(55-87)$ & $69.9 \pm 8.37(58-92)$ & 0.60 \\
\hline $\mathrm{eGFR}\left[\mathrm{ml} / \mathrm{min} / \mathrm{m}^{2}\right]$ & $83.7 \pm 23.9(44-151)$ & $86.7 \pm 23.2(49-156)$ & 0.44 \\
\hline \multicolumn{4}{|c|}{ Serum lipid profile [mg/dl]: } \\
\hline Total cholesterol & $210.8 \pm 46.7(148-373)$ & $193.9 \pm 43.6(119-257)$ & 0.38 \\
\hline LDL & $136.8 \pm 36.2(64-244)$ & $125.1 \pm 34.5(32-210)$ & 0.11 \\
\hline Triglycerides & $190.9 \pm 83.3(54-523)$ & $184.7 \pm 82.7(67-449)$ & 0.68 \\
\hline $\mathrm{HDL}$ & $42.9 \pm 13.6(24-121)$ & $43.3 \pm 12.9(23-72)$ & 0.67 \\
\hline \multicolumn{4}{|c|}{ Medical treatment, $n(\%)$ : } \\
\hline ACEI & $27(29)$ & $20(42)$ & 0.14 \\
\hline ARB & $18(20)$ & $8(17)$ & 0.67 \\
\hline$\beta$-blockers & $38(41)$ & $16(33)$ & 0.35 \\
\hline $\mathrm{CCB}$ & $16(17)$ & $7(15)$ & 0.67 \\
\hline Statin & $38(41)$ & $23(48)$ & 0.45 \\
\hline \multicolumn{4}{|l|}{ Serum biomarkers: } \\
\hline CRP $[\mathrm{mg} / \mathrm{dl}]$ & $4.84 \pm 4.3(0.1-19.3)$ & $4.24 \pm 3.84(0.3-17.6)$ & 0.51 \\
\hline
\end{tabular}

$B M I$ - body mass index, SBP - systolic blood pressure, DBP - diastolic blood pressure, eGFR - estimated glomerular filtration rate, ACEI angiotensin converting enzyme inhibitor, $A R B$ - angiotensin receptor blocker, CCB - calcium channel blocker, 25-OHD-25-hydroxyvitamin D.

In this study, we found no significant association between VD status and LVEF and LV diastolic function parameters (E, E', DT, IVRT, MPI). There was a significant association between VD deficiency and impaired GLS and GLSR in both diabetic and non-diabetic groups versus the control group. Notably, this association was independent of the broad spectrum of potential confounders including classical risk factors. That means that VD deficiency was independently associated with impaired LV GLS and GLSR and it is reasonable to think that VD deficiency can actually lead to myocardial dysfunction independently. VD likely affects systolic longitudinal motions initially before diastolic functions in the LV.

We also determined that 2D STE was better than conventional echocardiographic parameters LVEF and fractional shortening (LVFS) in detecting myocardial function in patients with VD deficien- cy. To our knowledge, this is the first study to investigate the association between VD deficiency and impaired subclinical myocardial dysfunction in diabetic and non-diabetic patients with normal coronary arteries [30].

Chen et al. studied diabetic patients with and without VD deficiency [31]. In this study, the patients had no coronary artery disease. They reported that diabetic patients with VD deficiency have impaired GLS versus diabetics with normal VD levels. In contrast, we included non-diabetic patients and the effect of VD on myocardial function and deformation parameters was also demonstrated. Also, the absence of coronary artery disease was confirmed by selective coronary artery angiography in diabetic and non-diabetic patients.

Sunbul et al. [32] studied 50 non-diabetic patients with VD deficiency who had no coronary artery disease. The evaluation of the coronary artery 
Pelin Karaca Ozer, Samim Emet, Ekrem Bilal Karaayvaz, Ali Elitok, Ahmet Kaya Bilge, Kamil Adalet, Aytac Oncul

Table III. Echocardiographic parameters of diabetic and non-diabetic patients

\begin{tabular}{|c|c|c|c|}
\hline Parameter & $\begin{array}{l}\text { Diabetic group } \\
\quad(n=80)\end{array}$ & $\begin{array}{l}\text { Non-diabetic group } \\
\quad(n=60)\end{array}$ & $P$-value \\
\hline LV EF (\%) & $68.18 \pm 4.79(58-78)$ & $68.28 \pm 5.44(55-77)$ & 0.72 \\
\hline IVST [mm] & $0.99 \pm 0.07(0.8-1.1)$ & $0.96 \pm 0.06(0.8-1.1)$ & 0.51 \\
\hline PWT [mm] & $0.91 \pm 0.08(0.7-1.1)$ & $0.88 \pm 0.08(0.7-1.0)$ & 0.52 \\
\hline LVEDV [ml] & $4.75 \pm 0.35(3.7-5.5)$ & $4.68 \pm 0.39(3.9-5.5)$ & 0.87 \\
\hline LVESV [ml] & $2.87 \pm 0.28(2.1-3.5)$ & $2.84 \pm 0.31(2.3-3.5)$ & 0.74 \\
\hline LVMI $\left[\mathrm{g} / \mathrm{m}^{2}\right]$ & $85.2 \pm 12.8(69.8-107.2)$ & $84.7 \pm 12.2(69.1-104.3)$ & 0.89 \\
\hline $\mathrm{E}[\mathrm{cm} / \mathrm{s}]$ & $65.5 \pm 20.9(25-110)$ & $79.4 \pm 23.2(37-124)$ & $<0.001$ \\
\hline $\mathrm{A}[\mathrm{cm} / \mathrm{s}]$ & $78.15 \pm 16.7(39-112)$ & $68.1 \pm 16.1(34-96)$ & $<0.001$ \\
\hline $\mathrm{E}^{\prime}[\mathrm{cm} / \mathrm{s}]$ & $6.4 \pm 2.39(2-12)$ & $8.8 \pm 2.54(3-13)$ & $<0.001$ \\
\hline $\mathrm{A}^{\prime}[\mathrm{cm} / \mathrm{s}]$ & $8.69 \pm 1.70(4-13)$ & $7.1 \pm 1.97(3-11)$ & $<0.001$ \\
\hline $\mathrm{DT}[\mathrm{cm} / \mathrm{s}]$ & $154.03 \pm 44.5(74-265)$ & $137.9 \pm 38.7(63-254)$ & 0.03 \\
\hline IVRT $[\mathrm{cm} / \mathrm{s}]$ & $108.35 \pm 18.5(64-146)$ & $95.03 \pm 15.7(63-137)$ & 0.01 \\
\hline GLS (\%) & $-15.31 \pm 3.88(-24-8)$ & $-16.68 \pm 3.01(-23-10)$ & 0.02 \\
\hline $\mathrm{SR}(\mathrm{s}-1)$ & $0.96 \pm 0.11(0.68-1.18)$ & $1.15 \pm 0.12(0.73-1.32)$ & 0.03 \\
\hline MPI (\%) & $0.71 \pm 0.9(0.32-1.07)$ & $0.45 \pm 0.11(0.32-0.83)$ & $<0.001$ \\
\hline
\end{tabular}

LV - left ventricular, EF- ejection fraction, IVST - interventricular septum thickness, PWT-posterior wall thickness, LVEDV - left ventricular end diastolic volume, LVESV - left ventricular end systolic volume, LVMI - left ventricular mass index, E - early diastolic velocity of mitral valve inflow, A - late diastolic velocity of mitral valve inflow, $E^{\prime}$ - early diastolic velocity at basal mitral annulus, $A^{\prime}-l a t e$ diastolic velocity at basal mitral annulus, DT - deceleration time, GLS - global longitudinal strain, SR - strain rate, MPI-myocardial performance index, IVRT - isovolumetric relaxation time.

Table IV. Echocardiographic parameters of patients with and without vitamin D deficiency

\begin{tabular}{|c|c|c|c|}
\hline Parameter & $\begin{array}{l}\text { VD deficiency group } \\
\qquad(n=92)\end{array}$ & $\begin{array}{l}\text { Group without VD deficiency } \\
\qquad(n=48)\end{array}$ & $P$-value \\
\hline LV EF (\%) & $68.6 \pm 5.07(55-78)$ & $67.3 \pm 4.98(58-77)$ & 0.13 \\
\hline IVST [mm] & $0.98 \pm 0.071(0.8-1.1)$ & $0.97 \pm 0.075(0.8-1.1)$ & 0.38 \\
\hline PWT $[\mathrm{mm}]$ & $0.90 \pm 0.078(0.7-1.0)$ & $0.89 \pm 0.096(0.7-1.1)$ & 0.42 \\
\hline LVEDV $[\mathrm{ml}]$ & $4.69 \pm 0.39(3.9-5.5)$ & $4.77 \pm 0.32(3.7-5.5)$ & 0.40 \\
\hline $\operatorname{LVESV}[\mathrm{ml}]$ & $2.84 \pm 0.29(2.1-3.4)$ & $2.90 \pm 0.29(2.1-3.5)$ & 0.38 \\
\hline LVMI $\left[\mathrm{g} / \mathrm{m}^{2}\right]$ & $85.0 \pm 13.9(69.1-107.2)$ & $84.7 \pm 13.3(69.7-104.3)$ & 0.78 \\
\hline $\mathrm{E}[\mathrm{cm} / \mathrm{s}]$ & $65.8 \pm 20.7(25-124)$ & $72.1 \pm 18.9(34-110)$ & 0.82 \\
\hline $\mathrm{A}[\mathrm{cm} / \mathrm{s}]$ & $70.7 \pm 17.3(38-112)$ & $74.5 \pm 15.0(34-110)$ & 0.62 \\
\hline $\mathrm{E}^{\prime}[\mathrm{cm} / \mathrm{s}]$ & $7.02 \pm 2.77(2-12)$ & $7.73 \pm 2.22(3-13)$ & 0.61 \\
\hline $\mathrm{A}^{\prime}[\mathrm{cm} / \mathrm{s}]$ & $7.38 \pm 1.86(3-13)$ & $7.58 \pm 1.79(4-11)$ & 0.45 \\
\hline DT $[\mathrm{cm} / \mathrm{s}]$ & $148.9 \pm 46.6(63-265)$ & $145.3 \pm 34.8(74-254)$ & 0.87 \\
\hline IVRT $[\mathrm{cm} / \mathrm{s}]$ & $103.7 \pm 17.1(63-146)$ & $99.3 \pm 21.3(64-137)$ & 0.67 \\
\hline GLS (\%) & $-14.68 \pm 3.2(-24-8)$ & $-18.23 \pm 3.01(-24-10)$ & $<0.001$ \\
\hline$S R(s-1)$ & $0.95 \pm 0.11(0.62-1.12)$ & $1.15 \pm 0.12(0.82-1.33)$ & 0.003 \\
\hline MPI (\%) & $0.57 \pm 0.13(0.32-0.84)$ & $0.52 \pm 0.17(0.32-1.07)$ & 0.34 \\
\hline
\end{tabular}

LV-left ventricular, EF- ejection fraction, IVST - interventricular septum thickness, PWT-posterior wall thickness, LVEDV - left ventricular end diastolic volume, LVESV - left ventricular end systolic volume, LVMI - left ventricular mass index, E - early diastolic velocity of mitral valve inflow, $A$ - late diastolic velocity of mitral valve inflow, $E$ - early diastolic velocity at basal mitral annulus, $A^{\prime}-l a t e$ diastolic velocity at basal mitral annulus, DT - deceleration time, GLS - global longitudinal strain, SR - strain rate, MPI-myocardial performance index, IVRT - isovolumetric relaxation time. 
disease was based only on the patients' history. All patients were treated with VD analogs. They noted an improvement in systolic longitudinal, radial and circumferential strains of the LV after therapy. These two studies support our study but there are some differences in study designs.

Another surprising result of our study was the significant negative correlation between VD and GLS in the group with VD deficiency $(r=-0.52623$, $p<0.001$ ); a statistically significant positive correlation was found between VD and GLS in the group without VD deficiency $(r=0.286465, p=$ $0.048)$. The range of VD should be $20-40 \mathrm{ng} / \mathrm{dl}$ for optimum myocardial longitudinal systolic strain values. This result suggests that myocardial function is suppressed when the VD exceeds a critical value and exaggerated replacement may negatively affect the myocardium.

Hypervitaminosis D occurs when pharmacologic doses of VD are consumed for prolonged periods of time or from a single megadose [33]. No study has yet investigated the effect of high dose VD on myocardial function. The toxic effects of high dose VD have been demonstrated in previous studies. One study examined the effects of VD and VD analogs and showed that exposure of human bone cells to VD in culture increases the cells' mRNA expression and activity and thus increases oxygen radical production. These authors suggested that potential cell damage is caused by an increase in lipid oxidation in the cell membrane or intracellular compartments due to oxidative stress [34]. Another study showed that VD exposure at high supraphysiological concentrations caused toxic effects on skeletal muscle cells that inhibited myogenesis in culture medium [35].

A previous study used scanning electron microscopy to examine the hearts of Wistar rats treated with a high dose of VD. Multifocal cardionecrosis was detected macroscopically in $70 \%$ of the rats on the $7^{\text {th }}$ day when the rats were in the acute phase of intoxication. To explain VD-induced cardionecrosis, they suggested that calcium-activated neutral proteinases may have contributed to the structural alteration of the extracellular matrix components [36, 37]. Nevertheless, the present study is the first to demonstrate that VD toxicity leads to myocardial damage.

This study has a relatively small sample size and is cross-sectional. Therefore, prospective investigations - possibly with randomization to VD supplementation - are warranted. Furthermore, serum VD levels may change throughout the day and across seasons. Thus, a single measurement may not reflect the actual VD status.

In conclusion, this study demonstrated that lower VD levels were significantly associated with impaired myocardial deformation parameters in diabetic and non-diabetic patients with nor-

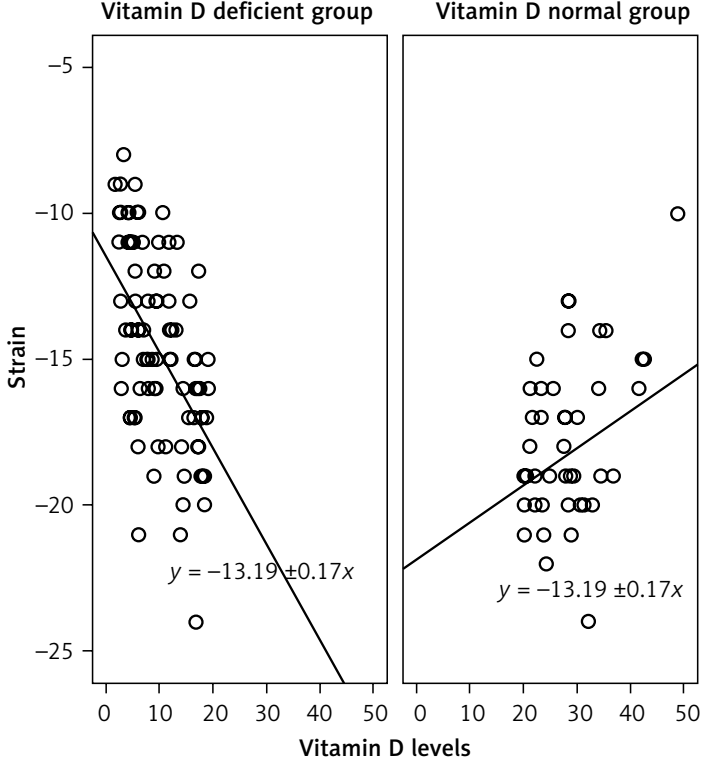

Figure 3. Correlation of VD levels and left ventricle global longitudinal strain in patients with vitamin $D$ deficiency and without vitamin $D$ deficiency

mal coronary arteries and preserved EF. Lower VD levels may play a role in the pathogenesis of myocardial dysfunction. VD deficiency is a pandemic disease but is rarely diagnosed and treated by physicians although it is easy and affordable to treat. The range of vitamin $D$ required for optimal myocardial longitudinal systolic strain values is $20-40 \mathrm{ng} / \mathrm{dl}$, and unnecessary replacement should be avoided.

\section{Conflict of interest}

The authors declare no conflict of interest.

\section{References}

1. Bikle D. Nonclassic actions of vitamin D. J Clin Endocrinol Metab 2009; 94: 26-34.

2. Hollis BW, Wagner $\mathrm{CL}$. The role of the parent compound vitamin $\mathrm{D}$ with respect to metabolism and function: Why clinical dose intervals can affect clinical outcomes. J Clin Endocrinol Metab 2013; 98: 4619-28.

3. Pike JW, Christakos S. Biology and mechanisms of action of the vitamin D hormone. Endocrinol Metab Clin North Am 2017; 46: 815-43.

4. Van Schoor N, Lips P. Global overview of vitamin D status. Endocrinol Metab Clin North Am 2017; 46: 845-70.

5. Wasson LT, Shimbo D, Rubin MR, Shaffer JA, Schwartz JE, Davidson KW. Is vitamin D deficiency a risk factor for ischemic heart disease in patients with established cardiovascular disease? 10-year follow-up of the Nova Scotia Health Survey. Int I Cardiol 2011; 148: 387-9.

6. Zittermann A, Schleithoff SS, et al. Putting cardiovascular disease and vitamin $D$ insufficiency into perspective. Br J Nutrition 2005; 94: 483-92.

7. Grübler MR, März W, Pilz S, et al. Vitamin D concentrations, cardiovascular risk and events - a review of epidemiological evidence. Rev Endocr Metab Disord 2017; 18: 259-72. 
8. Al Mheid I, Quyyumi AA. Vitamin D and cardiovascular disease: controversy unresolved. J Am Coll Cardiol 2017; 70: 89-100.

9. Rai V, Agrawal DK. Role of vitamin D in cardiovascular diseases. Endocrinol Metab Clin North Am 2017; 46 1039-59.

10. Norman PE, Powell JT. Vitamin D and cardiovascular disease. Circ Res 2014; 114: 379-93.

11. Wang L, Manson JE, Song Y, Sesso HD. Systematic review: vitamin $D$ and calcium supplementation in prevention of cardiovascular events. Ann Inter Med 2010 152: 315-23.

12. Potter E, Marwick TH. Assessment of left ventricular function by echocardiography: the case for routinely adding global longitudinal strain to ejection fraction. JACC Cardiovasc Imaging. 2018; 11: 260-74.

13. Holick MF, Binkley NC, Bischoff-Ferrari HA, et al. Evaluation, treatment, and prevention of vitamin D deficiency: an Endocrine Society clinical practice guideline. J Clin Endocrinol Metab 2011; 96: 1911-30.

14. Lang RM, Badano LP, MorAvi V, et al. Recommendations for cardiac chamber quantification by echocardiography in adults: an update from the American Society of Echocardiography and the European Association of Cardiovascular Imaging. Eur Heart J Cardiovasc Imaging 2015; 16: 233-70.

15. Nagueh SF. Left ventricular diastolic function. understanding pathophysiology, diagnosis, and prognosis with echocardiography. JACC: Cardiovascular Imaging 2020; 13: 228-44.

16. Feigenbaum H, Armstrong WF, Ayan T. Feigenbaum's Echocardiography. $7^{\text {th }}$ ed. Lippincotts Williams\&Wilkins, Philadelphia 2011

17. Marwick TH, Leano RL, Brown J, et al. Myocardial strain measurement with 2-dimensional speckle-tracking echocardiography: definition of normal range. JACC Cardiovasc Imaging 2009; 2: 80-4.

18. Clinical applications - speckle tracking. EACVI 3D Echocardiography Box.

19. Imga NN, Berker D, Can B, Guler S. The effects of three regimens of cholecalciferol (vitamin D3) supplementation on vitamin $D$ deficiency in non-obese and obese. Arch Med Sci Atheroscler Dis 2018; 3: e60-7.

20. Dziedzic E, Gąsior J, Pawłowski M. Vitamin D level is associated with severity of coronary artery atherosclerosis and incidence of acute coronary syndromes in non-diabetic cardiac patients. Arch Med Sci 2019; 15: 359-68.

21. Dziedzic EA, Gąsior JS, Pawłowski M, et al. Vitamin D level is associated with severity of coronary artery atherosclerosis and incidence of acute coronary syndromes in non-diabetic cardiac patients. Arch Med Sci 2019; 15: 359-68.

22. Elamin MB, Abu ENO, Elamin KB, et al. Vitamin D and cardiovascular outcomes: a systematic review and meta-analysis. J Clin Endocrinol Metab 2011; 96: 1931.

23. Griz LHM, Bandeira F, Gabbay MAL, Atala Dib S, de Carvalho EF. Vitamin D and diabetes mellitus: an update 2013. Arq Bras Endocrinol Metab 2014; 58: 1-8.

24. Ertek S, Akgül E, Cicero A, Kütük U. 25-Hydroxy vitamin $D$ levels and endothelial vasodilator function in normotensive women. Arch Med Sci 2012; 8: 47-52.

25. Norman PE, Powell JT. Vitamin D and cardiovascular disease. Circ Res 2014; 114: 379-93.

26. Somjen D, Weisman Y, Kohen F, et al. 25-hydroxyvitamin D3-1alpha-hydroxylase is expressed in human vascular smooth muscle cells and is upregulated by parathyroid hormone and estrogenic compounds. Circulation 2005 111: 1666-71.

27. Li YC, Kong J, Wei M, Chen ZF, Liu SO, Cao LP. 1,25-Dihydroxyvitamin $\mathrm{D}(3)$ is a negative endocrine regulator of the renin-angiotensin system. J Clin Invest 2002; 110 229-3

28. Bugger $\mathrm{H}$, Abel ED. Molecular mechanisms of diabetic cardiomyopathy. Diabetologia 2014; 57: 660-71.

29. Tsioufis C, Bafakis I, Kasiakogias A, et al. The role of matrix metalloproteinases in diabetes mellitus. Curr Top Med Chem 2012; 12: 1159-65.

30. Nikooyeh B, Neyestani TR. Oxidative stress, type 2 diabetes and vitamin D: past, present and future. Diabetes Metab Res Rev 2016; 32: 260-7.

31. Chen Y, Zhao CT, Zhen Z, Wong A, Tse HF, Yiu KH. Association of myocardial dysfunction with vitamin $D$ deficiency in patients with type 2 diabetes mellitus. J Diabetes Complications 2014; 28: 286-90.

32. Sunbul M, Bozbay M, Mammadov C, et al. Effect of vitamin D deficiency and supplementation on myocardial deformation parameters and epicardial fat thickness in patients free of cardiovascular risk. Int J Cardiovasc Imaging 2015; 31: 765-72.

33. Vieth, R. Vitamin D supplementation, 25 hydroxy-vitamin D concentrations, and safety. Am J Clin Nutr 1999; 69: 842-56.

34. Somjen D, Katzburg S, Knoll E, Sharon O, Posner GH, Stern N. Vitamin D analogs induce lipoxygenase mRNA expression and activity as well as reactive oxygen species (ROS) production in human bone cells. J Steroid Biochem Mol Biol 2010; 121: 265-7.

35. Ryan KJ, Daniel ZC, Craggs LJ, Parr T, Brameld JM Dose-dependent effects of vitamin D on transdifferentiation of skeletal muscle cells to adipose cells. J Endocrinol 2013; 217: 45-58.

36. Walentynowicz O, Kubasik-Juraniec J, Rudzińska-Kisiel T. Myocardial necrosis due to vitamin D3 overdose: scanning electron microscopic observations. Folia Morphol 2004; 63: 439-44

37. Wrzołkowa T, Rudzińska-Kisiel T, Kłosowska B. Calcium content of serum and myocardium in vitamin D-induced cardionecrosis. Bone Miner 1991; 13: 111-21. 